\title{
Correlated electrons in a zig-zag chain with the spin-orbit interaction: Exact solution
}

\author{
A.A. Zvyagin \\ B. Verkin Institute for Low Temperature Physics and Engineering of the National Academy of Sciences of Ukraine \\ 47 Nauky Ave., Kharkiv 61103, Ukraine \\ V.N. Karazin Kharkiv National University, 4 Svobody sq., Kharkiv 61002, Ukraine \\ Max-Planck-Institut für Physik komplexer Systeme, 38 Nöthnitzer Str., D-01187, Dresden, Germany \\ E-mail: zvyagin@ilt.kharkov.ua
}

Received May 15, 2018, published online October 26, 2018

\begin{abstract}
The correlated electron model on a geometrically frustrated one-dimensional lattice with the spin-orbit coupling is studied. The exact solution is obtained using the Bethe ansatz. Zig-zag interaction can produce incommensurate charge and spin structures for large enough frustrating interactions. The spin-orbit coupling yields the behavior of correlation functions, reminiscent of the Fulde-Ferrell-Larkin-Ovchinnikov (FFLO) features of real type-II superconductors.
\end{abstract}

Keywords: integrable correlated electron model, geometrical frustration, spin-orbit coupling.

\section{Introduction}

There has been recently considerable interest in quasione dimensional quantum correlated electron systems. They manifest specific features of the low-temperature behavior, absent in standard electron systems. The nature of those specific features is in the enhancement of quantum and thermal fluctuations of one-dimensional systems due to the peculiarities of their density of states [1]. Fluctuations usually destroy any order at nonzero temperatures there. However strong electron-electron correlations exhibit power-law decays in the ground state of such interacting systems. Therefore, such powerful methods of theoretical physics as perturbation theories and mean-field approximations are hardly applicable to strongly correlated one-dimensional systems. Namely because of that lowdimensional correlated electron systems are the best known examples in which non-perturbative methods like the renormalization group theory, Bethe's ansatz, bosonization, conformal field theory, etc. have manifested their advantages. The advantage of theoretical studies of onedimensional systems is the possibility to obtain exact solutions by using non-perturbative approaches, which are difficult to apply for higher-dimensional quantum many-body models [2]. The results of exact calculations of onedimensional models can serve as a testing ground for the use of perturbative and numerical methods in more realistic situations.
The interest in the behavior of electron and spin models on geometrically frustrated lattices [3] is related to the possibility to find there, due to the frustration, the emergent excitations with the fractional charges and spins, like in quantum spin liquids [4]. For theoretical description in the frustrated case it is also very hard to use standard mean field or perturbative schemes of calculations, successfully used for electron systems without frustration. Here rare examples of exactly solvable models on frustrated lattices also are very important. Zig-zag quantum chains have attracted interest of physicists due to various aspects of their characteristics. For example, zig-zag spin chains reveal many interesting properties, including possible quantum phase transitions to incommensurate phases [5], graphene nanoribbons [6], superconductivity in zig-zag chains [7], plasmonic systems [8], and ultracold atoms [9] on zig-zag lattices. Zero-energy Majorana edge states were observed in magnetic iron zig-zag chains on the surface of superconducting lead using spectroscopic imaging technique [10], and, in zig-zag edge states for Bi bilayers [11]. Excitations in the bulk of chains were gapped due to the proximity effect of the s-wave superconducting lead substrate. Such co-existence of the one-dimensionality, pairing and geometrically frustrated lattices also motivated our study. On the other hand, the Ising pairing anisotropy has been recently observed in $\mathrm{NbSe}_{2}$ atomic layers [12], which motivated our work, too. 
A spin-orbit (SO) interaction manifests itself as the effect of an electric field on a moving charged particle with spin. It is of special importance in spintronics, where the spin of electrons in electronic devices is manipulated and detected. In low-dimensions, the semiconductor device structure may give rise to an internal electric field and hence a SO coupling of the Rashba or Dresselhaus type [13]. It is important to investigate the effects of the SO coupling together with the interactions between particles. The SO coupling can be also caused in correlated electron chains by strains of the lattice [14]. Recently systems where the SO interactions plays the crucial role in low-dimensional electron systems, like edge or surface states of topological insulators [15] or semiconductor nanowires [16], have attracted the attention of researchers. For instance, ultracold atoms in optical lattices [17] offer a great opportunity to investigate many body quantum phenomena due to the very good experimental control over the density of atoms, the purity of the system, and inter-atomic interactions [18]. These systems can be prepared almost free of impurities, the interaction strength can be tuned by external parameters and the very low temperatures enable experimentalists to study the various quantum phases. For low-dimensional systems the properties are very close to those of models, for which many exact results have been obtained [19]. Spin-imbalanced ultracold gases of atoms confined to one-dimensional optical traps have been the subject of many recent studies. The transverse harmonic confinement of the atoms leads to a confinement-induced Feshbach-type resonance, which can be tuned to give rise to an attractive local interaction whose strength can be varied [20]. The confinement along the tube is weak and roughly harmonic and in theoretical considerations it can be incorporated into the chemical potential of the atoms, giving rise to phase separation [19]. Fermionic ultracold atoms with a local inter-atomic interaction and spin imbalance can be described by Bethe ansatz solvable models [21]. Recently both, Abelian and non-Abelian gauge fields, have been experimentally realized for ultracold atomic bosonic [22] and fermionic [23] gases using two-photon Raman processes. In particular, the non-Abelian gauge field leads to an effective SO interaction for ultracold atoms that can be considered effective spin- $1 / 2$ fermions. In the studied ${ }^{40} \mathrm{~K}$ system, the two spin-1/2 states are chosen as the two magnetic sublevels with $|\uparrow\rangle=|9 / 2,9 / 2\rangle$ and $|\downarrow\rangle=|9 / 2,7 / 2\rangle$ (or alternatively the two sublevels can be chosen as the second and third lowest hyperfine levels in ${ }^{6} \mathrm{Li}$ ). Disregarding other levels, the studied system is effectively a spin-1/2 fermion system. In the experiments [23] these states are coupled by a pair of Raman beams. The resulting artificial SO interaction has induced spin dephasing in the quantum spin dynamics of the ultracold fermions.

In the present study we find the solution to the new Bethe ansatz integrable model of interacting electrons on the one-dimensional lattice with nearest- $(\mathrm{NN})$ and nextnearest neighbor (NNN) hoppings (equivalent to the zig- zag lattice) and SO interactions. The Ising type of the anisotropy gives rise to a gap for low-energy unbound electron states at small values of the magnetic field. The model is reminiscent to the type-II superconductor, because in that region of applied field only spin-singlet Cooper-like pairs are gapless; for larger values of the field both pairs and unbound electron states are gapless, and, finally, for larger field the system is in the spin-polarized phase. For the large value of the parameter, which describes the interchain or NNN hoppings and couplings, the model manifests additional quantum phase transitions to phases with inhomogeneously distributed charge and/or spin densities. At low temperature, as usually, quantum phase transitions reveal themselves as square root features in the behavior of the magnetic and charge susceptibility and the specific heat. The SO coupling manifests itself in the finite size corrections, important for asymptotic behavior of correlation functions.

\section{The considered model}

Let us start with the consideration of a one-dimensional lattice with spin- $1 / 2$ electrons and SO interaction

$$
\begin{aligned}
\mathcal{H}_{0} & =-\sum_{j \sigma}\left[t^{\prime}\left(\psi_{j+1, \sigma}^{\dagger} \psi_{j, \sigma}+\psi_{j, \sigma}^{\dagger} \psi_{j+1, \sigma}\right)+\right. \\
& \left.+i g \sigma\left(\psi_{j+1, \sigma}^{\dagger} \psi_{j, \sigma}-\psi_{j, \sigma}^{\dagger} \psi_{j+1, \sigma}\right)\right],
\end{aligned}
$$

where $\psi_{j, \sigma}^{\dagger}$ creates the electron at site $j$ with the spin projection $\sigma=\uparrow, \downarrow, t^{\prime}$ is the hopping integral, and $g$ the SO interaction parameter. The terms of the Hamiltonian can be combined into an effective complex hopping parameter [24]

$$
\mathcal{H}_{0}=-\sum_{j \sigma}\left(t \psi_{j+1, \sigma}^{\dagger} \Psi_{j, \sigma}+\text { H.c. }\right)
$$

where $t=t^{\prime}+i g \sigma=\sqrt{t^{\prime 2}+g^{2} / 4} \exp (i 2 \pi \sigma \phi)$ and the phase factor $\phi=(1 / \pi) \arctan \left(g / 2 t^{\prime}\right)$ is caused by the SO interaction. A gauge transformation removes the phase factor from the Hamiltonian for the open chain, or transfers it into a spin-dependent twisted boundary condition for the ring. Such a gauge transformation can be performed also for the case in which double occupation of each site is excluded with the hopping term replaced by $\mathcal{H}_{0}=\sum_{j} \mathcal{H}_{j, j+1}^{0}$, where

$$
\mathcal{H}_{j, j+1}=-\sum_{\sigma} \mathcal{P}_{j}\left(t \psi_{j+1, \sigma}^{\dagger} \psi_{j, \sigma}+\text { H.c. }\right) \mathcal{P}_{j}
$$

where $\mathcal{P}_{j}=\left(1-n_{j,-\sigma}\right)\left(1-n_{j+1,-\sigma}\right) \quad\left(\right.$ with $\left.n_{j, \sigma} \equiv \psi_{j, \sigma}^{\dagger} \psi_{j, \sigma}\right)$ is the projection operator which excludes the double occupation at each site. Here we propose the new exactly solvable correlated electron model on the zig-zag onedimensional lattice with the following interaction 


$$
\begin{gathered}
\mathcal{H}=t \sum_{j}\left(\mathcal{H}_{j, j+2}+\frac{\sinh ^{2}(\eta)}{\sin ^{2}(\theta)+\sinh ^{2}(\eta)}\left(-\mathcal{H}_{j, j+2}+\right.\right. \\
\left.\left.+\hat{B}\left(\mathcal{H}_{j, j+1}+\mathcal{H}_{j+1, j+2}\right)-i \frac{\tan (\theta)}{\sinh (\eta)}\left[\mathcal{H}_{j, j+1}, \mathcal{H}_{j+1, j+2}\right]\right)\right),
\end{gathered}
$$

where

$$
\begin{aligned}
& \mathcal{H}_{j, j+1}=-\sum_{\sigma} \mathcal{P}_{j}\left(\psi_{j+1, \sigma}^{\dagger} \psi_{j, \sigma}+\text { H.c. }\right) \mathcal{P}_{j}+ \\
& +\left(\psi_{j, \downarrow}^{\dagger} \psi_{j+1, \uparrow} \psi_{j+1, \uparrow}^{\dagger} \psi_{j, \downarrow}+\psi_{j, \uparrow}^{\dagger} \psi_{j+1, \downarrow} \psi_{j+1, \downarrow}^{\dagger} \psi_{j, \uparrow}\right)-
\end{aligned}
$$

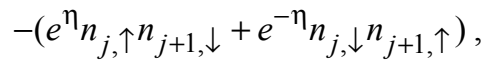

and [.,.] denotes the commutator. The operator $\hat{B}$ modifies the hopping and the transverse interaction amplitudes by the factor $\cos (\theta)$. The model is the generalization of the anisotropic [25] supersymmetric $t-J$ model (with $J=2 t$ ) [26] (the parameter $\eta$ describes the "easy-axis" magnetic anisotropy) for the case of the NN and the NNN interactions and hoppings with the SO coupling. The chain with the NN and NNN bonds is equivalent to the zig-zag chain. The parameter $\theta$ is responsible for the inclusion of the NNN connections. Obviously for $\theta=0$ we obtain the Hamiltonian of the anisotropic supersymmetric $t-J$ chain with only NN hoppings and couplings. The three-site terms in the Hamiltonian in the second line in Eq. (4) violate the time-reversal and parity symmetries separately, while the Hamiltonian is, naturally, invariant with respect to the product of those symmetries. Those terms are total time derivatives in the classical sense (i.e., they do not change the classical equations of motion for the classical counterpart of the considered system), and they are only important in the quantum mechanical aspects. In the limit $\eta \rightarrow 0$ the Hamiltonian is reduced to the $s u(1 \mid 2)$-symmetric $t-J$ model with the SO coupling (which, naturally, distinguishes the direction $z$ itself). It can be seen by the rescaling of the Hamiltonian $\theta \rightarrow \eta \theta^{\prime}$ with the finite $\theta^{\prime}$ before taking the limit $\eta \rightarrow 0$. Notice also that the Hamiltonian is periodic in $\theta$. In the limiting case $\eta=0$ and $\theta=0$ the Hamiltonian is reduced to the $s u(1 \mid 2)$-symmetric $t-J$ model with the SO coupling [27]. On the other hand, for $\eta=0$ and $\phi=0$ the model describes the limiting case of $N=2$ coupled supersymmetric $t-J$ chains [28].

The model remains exactly solvable if we add to the Hamiltonian $\mathcal{H}$ the Zeeman term and the term with the chemical potential $\mu^{\prime}$ (for the grand canonical ensemble)

$$
\mathcal{H}_{l}=-\sum_{j}\left(\frac{H^{\prime}}{2}\left(n_{j, \uparrow}-n_{j, \downarrow}\right)+\mu^{\prime} \sum_{\sigma} n_{j, \sigma}\right),
$$

where $H^{\prime}$ is the external magnetic field (the model is exactly solvable only if the field is directed along the axis of the magnetic anisotropy and the direction, distinguished by the SO interaction). The SO coupling manifests itself twofold: First, it (trivially) renormalizes the common multiplier, and, second, it produces the phase shift $\phi$. Below we concentrate on the nontrivial effect of the SO-caused phase shift. Hence, in what follows we put $t=1$, and renormalize the values of the chemical potential $\mu^{\prime} \rightarrow t \mu$ and $H^{\prime} \rightarrow t H$ for simplicity.

\section{The Bethe ansatz}

Using the algebraic Bethe ansatz method described in Ref. 2 we can prove that the Hamiltonian $\mathcal{H}+\mathcal{H}_{l}$ is diagonalized by Bethe's ansatz in terms of two sets of parameters, $\left\{v_{j}\right\}_{j=1}^{N}$ and $\left\{\Lambda_{\alpha}\right\}_{\alpha=1}^{M}$, with $N$ being the number of electrons, and $M$ being the number of spin down electrons. Those parameters are called rapidities; they describe unbound electrons and spinons, respectively. It can be shown that the ground state is described by $N-2 M$ unbound electron states with the real rapidities $v_{j}$ and $M$ spin-singlet Cooper-like pairs (bound states) for which charge rapidities are complex conjugated pairs [25] To the exponential accuracy $\exp (-L)$, where $2 L$ is the length of the system, the rapidities of pairs can be written as $v_{\alpha}=\Lambda_{\alpha} \pm i \eta / 2$. The rapidities satisfy the following Bethe ansatz equations

$$
\begin{gathered}
\left(\frac{\sin \left(v_{j}+i \eta / 2\right)}{\sin \left(v_{j}-i \eta / 2\right)} \frac{\sin \left(v_{j}-\theta+i \eta / 2\right)}{\sin \left(v_{j}-\theta-i \eta\right) / 2}\right)^{L}= \\
=\mathrm{e}^{-i \pi \phi} \prod_{\beta=1}^{M} \frac{\sin \left(v_{j}-\Lambda_{\beta}+i \eta / 2\right)}{\sin \left(v_{j}-\Lambda_{\beta}-i \eta / 2\right)}, \\
=-\left(\frac{\sin \left(\Lambda_{\alpha}-v_{j}+i \eta\right)}{\sin \left(\Lambda_{\alpha}-v_{j}-i \eta\right)} \frac{\sin \left(\Lambda_{\alpha}-v_{j}-\theta+i \eta\right)}{\sin \left(\Lambda_{\alpha}-v_{j}-\theta-i \eta\right)}\right)^{L}= \\
\prod_{k=1}^{N-2 M} \frac{\sin \left(\Lambda_{\alpha}-v_{k}+i \eta / 2\right)}{\sin \left(\Lambda_{\alpha}-v_{k}+i \eta / 2\right)} \frac{\sin \left(\Lambda_{\alpha}-v_{k}-\theta+i \eta / 2\right)}{\sin \left(\Lambda_{\alpha}-v_{k}-\theta-i \eta / 2\right)} \times \\
\times \prod_{\beta=1}^{M} \frac{\sin \left(\Lambda_{\alpha}-\Lambda_{\beta}+i \eta\right)}{\sin \left(\Lambda_{\alpha}-\Lambda_{\beta}-i \eta\right)} .
\end{gathered}
$$

The number of particles $N$ and the magnetization $N / 2-M$ are controlled by the chemical potential and the external magnetic field, respectively. The energy the state is given by

$$
\begin{gathered}
E=-2 \sum_{j=1}^{N-2 M}\left(\frac{1-\cos \left(2 v_{j}\right) \cosh (\eta)}{\cosh (\eta)-\cos \left(2 v_{j}\right)} \frac{1-\cos \left(2 v_{j}-2 \theta\right) \cosh (\eta)}{\cosh (\eta)-\cos \left(2 v_{j}-2 \theta\right)}\right)- \\
-\frac{H}{2}(N-2 M)-2 \cosh (\eta) \sum_{\alpha=1}^{M}\left(4-\frac{\sinh ^{2}(\eta)}{\sin ^{2}\left(\lambda_{\alpha}\right)+\sinh ^{2}(\eta)}-\right. \\
\left.-\frac{\sinh ^{2}(\eta)}{\sin ^{2}\left(\lambda_{\alpha}-\theta\right)+\sinh ^{2}(\eta)}\right)-\mu N .
\end{gathered}
$$


We also see that the Bethe ansatz equations are periodic in $\theta$, i.e. it is enough to consider $\theta$ in the domain $-\pi \leq \theta \leq \pi$.

Notice that equations (7) are written for the ring geometry. For the open chain they have similar structure with standing waves instead of plane waves for the ring, with the SO interaction-caused phase factor trivially removed by a gauge transformation, and, hence, in what follows we consider the more generic case of the ring geometry.

\section{The ground state}

The ground state is organized by the filling of Fermi seas (i.e. states with negative energies) with $N-2 M$ unbound electron states with real $v_{j}$ rapidities, and $M$ Cooper-like pairs with complex conjugated rapidities. In the thermodynamic limit $L, N, M \rightarrow \infty$ with fixed ratios $N / L$ and $M / L$ the ground state is given by the solution of two Fredholm integral equations for the density of unbound electron states $\rho(v)\left(\rho_{h}(v)\right.$ is the density of the holes) and density of pairs $\sigma^{\prime}(\Lambda)\left(\sigma_{h}^{\prime}(\lambda)\right.$ is the density of holes for pairs)

$$
\begin{aligned}
& 2 \pi\left[\rho_{h}(v)+\rho(v)\right]=f(v, \eta / 2)+f(v-\theta, \eta / 2)- \\
& -\int d \Lambda f(v-\Lambda, \eta / 2) \sigma(\Lambda), \\
& 2 \pi\left[\sigma^{\prime}(\Lambda)+\sigma^{\prime}(\Lambda)\right]=f(\Lambda, \eta)+f(\Lambda-\theta, \eta)- \\
& -\int d z f(\Lambda-z, \eta) \sigma(z)-\int d v f(\Lambda-v, \eta / 2) \rho(v),
\end{aligned}
$$

where $f(x, y)=2 \sinh (2 y) /[\cosh (2 y)-\cos (2 x)$. The integrations in Eqs. (9) is over the values of $\Lambda, v$, and $z$, for which energies of states are negative, see below. In the thermodynamic the energy of the system is

$$
\begin{gathered}
\varepsilon_{\infty}=-2 \int d v \rho(v)\left(\frac{1-\cos (2 v) \cosh (\eta)}{\cosh (\eta)-\cos (2 v)}+\right. \\
\left.+\frac{1-\cos (2 v-2 \theta) \cosh (\eta)}{\cosh (\eta)-\cos (2 v-2 \theta)}\right)- \\
-2 \cosh (\eta) \int d \Lambda \sigma(\Lambda)\left(4-\frac{\sinh ^{2}(\eta)}{\sin ^{2}(\Lambda)+\sinh ^{2}(\eta)}-\right. \\
\left.-\frac{\sinh ^{2}(\eta)}{\sin ^{2}(\Lambda-\theta)+\sinh ^{2}(\eta)}\right)
\end{gathered}
$$

We can introduce the "dressed" energies for unpaired electron states $\varepsilon(v)$ and pairs $\Psi(\Lambda)$, which are determined from the following set of integral equations,

$$
\begin{aligned}
\varepsilon(k)= & f(v, \eta / 2)+f(v-\theta, \eta / 2)-\mu-\frac{H}{2}- \\
& -\frac{1}{2 \pi} \int d \Lambda \Psi(\Lambda) f(v-\Lambda, \eta / 2),
\end{aligned}
$$

$$
\begin{gathered}
\Psi(\Lambda)=f(\Lambda, \eta)+f(\Lambda-\theta, \eta)-2 \mu- \\
-\frac{1}{2 \pi} \int d z f(\Lambda-z, \eta) \Psi(z)-\frac{1}{2 \pi} \int d v f(\Lambda-v, \eta / 2) \varepsilon(v)
\end{gathered}
$$

We see that unbound electron states carry spin $1 / 2$ and the charge $-e$, while the Cooper-like singlet pairs carry zero spin and the charge $-2 e$. We also see that the SOinduced phase factor $\phi$ does not enter the characteristics of the system in the thermodynamic limit in the ground state; it manifests itself in the finite size corrections [2], see below.

Investigating Eqs. (11) we see that for the external magnetic fields $H<H_{c}$ only paired states are populated, where

$$
\begin{gathered}
H_{c}=-2 \mu+2 f(\pi, \eta / 2)+2 f(\pi-\theta, \eta / 2)- \\
-\frac{1}{\pi} \int d \Lambda f(\pi-\Lambda, \eta / 2) \Psi(\Lambda)
\end{gathered}
$$

The energies of unbound electron states are gapped. We can say that $H_{c}$ is one-half of the minimal external magnetic field necessary to depair the Cooper-like singlet pair state.

On the other hand, if the magnetic field is larger than $H_{s}$ the magnetization is saturated (it is equal to $N / 2$ ), and only unbound electron states are populated, where

$$
H_{s}=-2 \mu+2 f(\pi, \eta / 2)+2 f(\pi-\theta, \eta / 2) .
$$

At those critical values of the field the system undergoes the second order quantum phase transitions. Such a behavior is reminiscent of the type-II superconductor in the external magnetic field, because for $H<H_{c}$ there are only Cooper-like pairs in the system; for $H_{c} \leq H \leq H_{s}$ pairs and unbound electron states coexist, and, finally, for $H>H_{s}$ there are only unbound electron states. We emphasize, however that in the one-dimensional electron system with short-range interactions there is no true superconducting order with off-diagonal long-range ordering. Instead, correlation functions of Cooper-like pairs and unbound electron states decay with power laws at long times or distances, see below.

Let us first concentrate on the region with $H<H_{c}$ (i.e., $2 M=N$ ), where only spin-singlet pairs exist (let us call this phase the gapped one). In this case the Bethe equations in the thermodynamic limit can be reduced to

$$
\begin{gathered}
2 \pi\left[\sigma^{\prime}(\Lambda)+\sigma^{\prime}(\Lambda)\right]=f(\Lambda, \eta)+f(\Lambda-\theta, \eta)- \\
-\int d z f(\Lambda-z, \eta) \sigma(z), \\
\Psi(\Lambda)=f(\Lambda, \eta)+f(\Lambda-\theta, \eta)-2 \mu- \\
-\frac{1}{2 \pi} \int d z f(\Lambda-z, \eta) \Psi(z) .
\end{gathered}
$$


It is easy to show that the quantum numbers fill the interval beginning from its edges for any $\eta$. On the other hand, for any nonzero $\theta$ the "bare" energy $\Psi^{0}(\Lambda)=f(\Lambda, \eta)+$ $+f(\Lambda-\theta, \eta)-2 \mu$ shows two- or four-minima behavior, depending on the value of the parameter $\theta$, see Fig. 1 for the "symmetrized" case $\Lambda \rightarrow \Lambda+\theta / 2$. It means that the filling of the Fermi seas depends on the value of the chemical potential (band filling). For $\mu=0$ the system is half-filled, $N=L$. At small $\theta$ for $\mu<\mu_{c 1}$ there are only two Fermi seas for singlet pairs. In this case the integration limits in Eqs. (14) are $\left[-\pi+\theta / 2,-\Lambda_{0}+\theta / 2\right]$ and $\left[\Lambda_{0}+\theta / 2, \pi+\theta / 2\right]$, where $\Lambda_{0}$ plays the role of the Fermi point $\left(\Psi\left( \pm \Lambda_{0}\right)=0\right)$. For small enough $\eta$ for $\mu>\mu_{c}$ two Fermi seas of singlet pairs are empty, see Fig. 1. On the other hand, at large enough $\theta$ we have four Fermi seas. Two of them are filled for $\mu \leq \mu_{c 1}$. For $\mu_{c 1} \leq \mu \leq \mu_{c 2}$, the filling of other two seas is non-total, see Fig. 1. We emphasize, however, that the filling of two or four Fermi seas in those regions of $\mu$ are not independent; they are determined by the one parameter, the chemical potential $\mu$. Finally, for $\mu>\mu_{c}$ the band is empty. At all critical values of the chemical potential (i.e., at the critical fillings of the band) the charge susceptibility (the compressibility) shows in the ground state the square-root in $\mu$ behavior. notice, on the other hand, at the critical value of $\theta$ at which four Fermi seas appear (unfortunately, it is impossible to find its value analytically), the compressibility as a function of the chemical potential shows $\left(\mu-\mu_{c}\right)^{-3 / 4}$ behavior. Several minima in the dispersion law for "pure" (and, hence, "dressed") energies imply the spatial distribution of the electron density (with possible incommensurate distributions, depending on $\mu, \theta$ and $\eta$ ). Hence, for $H<H_{c}$ for

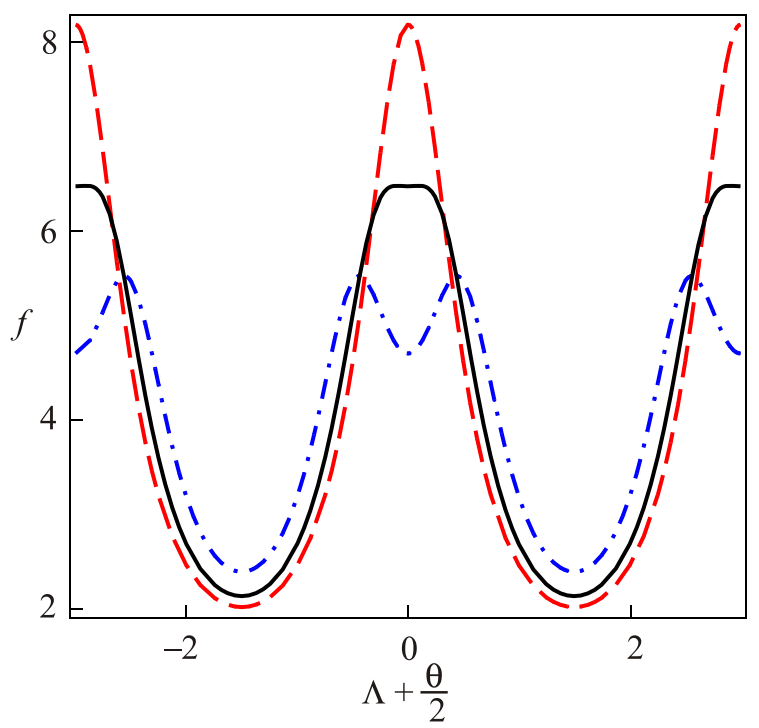

Fig. 1. (Color online) The dependence of the symmetrized case $\Lambda \rightarrow \Lambda+\theta / 2$ for "pure" energy of spin-singlet pairs for the gapped phase at $\eta=0.5$ for $\mu=0$. The dashed red line: $\theta$ small; the dashed-dotted blue line: $\theta$ large; the solid black line: the critical value of $\theta$. some values of the chemical potential we deal with the analogy of the CDW (charge density wave) commensurate distribution of electron density, but for Cooper-like singlet pairs not for electrons as in the standard CDW, and for others values the incommensurate distributions of the density of pairs can exist.

For $H>H_{s}$ there are no bound states, and

$$
\varepsilon(v)=f(v, \eta / 2)+f(v-\theta, \eta / 2)-\mu-(H / 2) .
$$

It means that in this region of parameters there is no "dressing" of the energies of unbound electron excitations due to the interactions.

Finally, for $H_{c} \leq H \leq H_{s}$ the situation is very complicated, because Fermi seas exist both for unbound electron states and for singlet pairs. Here, depending on the values of the chemical potential and magnetic field with respect to given $\theta$ and $\eta$ the system can manifest the spatial distribution of the density of spin-singlet pairs and unbound electron excitations [29]. For commensurate spatial distributions the latter imply the existence of SDW (spin density wave) like and CDW-like states; for other values of governing parameters incommensurate distributions of the spin and charge densities is expected. Again, depending on the values of $\theta$ and $\eta$ there can exist several critical values of the chemical potential and the magnetic field, at which second order quantum phase transitions with the square-root singularities in $\mu$ and $H$, respectively, take place. Also, as in the gapped phase, at the critical value of $\theta$ those singularities manifest $-3 / 4$ features.

\section{Correlation functions}

According to the conformal field theory [30] in the ground state correlation functions of states with gapless excitations decay with distance or with time algebraically [2] as $\sum_{j=u, p} \exp \left(-2 i D_{j} p_{j}^{F}\right)\left(x \pm i v_{j} t\right)^{-\theta}$ with all kinds of gapless states contributing, where $v_{j}$ is the Fermi velocity of unbound electron excitations or pairs, and $j=u, p$ mean unbound electron states and spin-singlet Cooper-like pairs. The exponents $\theta_{j}$ are determined from the conformal dimensions, see below.

For the asymptotes of correlation functions we need to find the finite size corrections to the ground state energy and the momentum, caused by low-energy excitations. For the simplest case of only two Fermi seas for unbound electron states and spin-singlet pairs (i.e., for the spatially homogeneous distributions of charge and spin densities in the ground state), are given by [31]

$$
E=L \varepsilon_{\infty}\left(\Lambda_{0}, v_{0}\right)+\sum_{l=u, p} \frac{\pi v_{l}}{2 L}\left(\sum_{q=u, p}\left(\hat{z}^{-1}\right)_{l, q} \Delta N_{q}\right)^{2}+
$$




$$
+\sum_{l=u, p} \frac{2 \pi v_{l}}{L}\left[\left(\sum_{q=u, p} z_{q, l} D_{q}\right)^{2}+n_{l}^{+}+n_{l}^{-}-\frac{1}{12}\right]
$$

where $v_{l}$ are the Fermi velocities

$$
v_{u}=\frac{d \varepsilon(v) / d v_{v=v_{0}}}{2 \pi \rho\left(v_{0}\right)}, \quad v_{p}=\frac{d \Psi(\Lambda) / d \Lambda_{\Lambda=\Lambda_{0}}}{2 \pi \sigma\left(\Lambda_{0}\right)},
$$

of the unpaired electron states and pairs, respectively, and

$$
\begin{gathered}
\Delta N_{u}=\Delta(N-2 M), \Delta N_{p}=\Delta M \\
D_{u}=\frac{1}{2}\left(\Delta N_{u}+\Delta N_{p}\right) \bmod 1, D_{p}=\frac{1}{2} \Delta N_{p} \bmod 1 .
\end{gathered}
$$

The expression for the momentum is

$$
\Delta P=\sum_{l=u, p}\left(\frac{2 \pi}{L}\left[D_{l} \Delta N_{l}+n_{l}^{+}-n_{l}^{-}\right]+2 p_{l}^{F} D_{l}\right)
$$

The quantum numbers $\Delta N_{u, p}$ refer to the change in the number of states in each Fermi sea by the low-energy excitation and $D_{u, p}$ are the corresponding backscattering quantum numbers (transfer from the left Fermi point to the right Fermi point). The number of particle-hole excitations about each Fermi point is denoted by $n_{u, p}^{ \pm}$. The backscattering quantum numbers for unbound electron states have to be shifted by the SO phase $D_{u} \rightarrow D_{u}-\phi$. Spin-singlet pairs are not affected by the SO coupling, naturally. The Fermi momenta corresponding to the Fermi seas are $p_{u}^{F}=\pi(N-2 M) / L$ and $p_{p}^{F}=\pi M / L$. The generalized dressed charges, $z_{l q}=\xi_{l q}\left(B_{q}\right)$, where $B_{q}=v_{0}$ and $B_{p}=\Lambda_{0}$, are obtained from the solution of the following set of integral equations $(l=u, p)$

$$
\begin{gathered}
\xi_{l u}(v)=\delta_{l, u}-\int d \Lambda f(v-\Lambda, \eta / 2) \xi_{l p}(\Lambda), \\
\xi_{l p}(\Lambda)=\delta_{l, p}-\int d z f(z-\Lambda, \eta) \xi_{l p}(z)- \\
-\int d v f(v-\Lambda, \eta / 2) \xi_{l u}(v) .
\end{gathered}
$$

Equations for the components of the dressed charge depend on $\theta$ via the limits of integration.

The conformal dimensions, which define the exponents of correlation functions, are related to the finite size corrections obtained above

$$
\begin{gathered}
\Delta_{u}^{ \pm}=n_{u}^{ \pm}+\left[z_{u u}\left(D_{u}-\phi\right)+z_{p u} D_{p} \pm\right. \\
\left. \pm\left(z_{p p} \Delta N_{u}-z_{u p} \Delta N_{p}\right) /(2 d)\right]^{2}, \\
\Delta_{p}^{ \pm}=n_{p}^{ \pm}+\left[z_{u p}\left(D_{u}-\phi\right)+z_{p p} D_{p} \pm\right. \\
\left. \pm\left(z_{u u} \Delta N_{p}-z_{p u} \Delta N_{u}\right) /(2 d)\right]^{2}
\end{gathered}
$$

in the mixed homogeneous phase (two Fermi seas, $\left.H_{c} \leq H \leq H_{s}\right)$. Here we denote $d=z_{p p} z_{u u}-z_{u p} z_{p u}$. The situation is similar to the Hubbard chain with attraction [32]. For the single electron (e.g., for $\sigma=\uparrow$ ) Green function in the homogeneous mixed phase the set of quantum numbers is the following: $\Delta N_{u}=1, \Delta N_{p}=0$, $n_{u, p}^{ \pm}=0$, and $D_{u}$ and $D_{p}$ are half-integers, with $D_{u}$ shifted by $\phi$. The two leading terms with the smallest exponents are for $D_{u}=-D_{p}= \pm 1 / 2$ and $D_{u}=D_{p}= \pm 1 / 2$, which we denote with indexes 1 and 2, respectively.

From now on let us concentrate, for simplicity, on the equal time correlation functions. For the single-electron (up-spin) Green function the exponents are $\theta_{j}=2\left(\Delta_{u}^{+}+\right.$ $\left.+\Delta_{u}^{-}+\Delta_{p}^{+}+\Delta_{p}^{-}\right)$. The equal-time asymptote of the singleelectron up-spin Green function is then

$$
\begin{gathered}
\left\langle\psi_{\uparrow}^{\dagger}(x, 0) \psi_{\uparrow}(0,0)\right\rangle \approx A_{1}\left(\frac{\mathrm{e}^{-i \varphi_{1}(\phi) x}}{x^{\theta_{1}(\phi)}}+\frac{\mathrm{e}^{i \varphi_{1}(-\phi) x}}{x^{\theta_{1}(-\phi)}}\right)+ \\
+A_{2}\left(\frac{\mathrm{e}^{-i \varphi_{2}(\phi) x}}{x^{\theta_{2}(\phi)}}+\frac{\mathrm{e}^{i \varphi_{2}(-\phi) x}}{x^{\theta_{2}(-\phi)}}\right),
\end{gathered}
$$

where the exponents and the wave numbers of the phases are

$$
\begin{gathered}
\theta_{1,2}(\phi)=\frac{1}{2}\left\{\left[z_{u u}(1-2 \phi) \mp z_{p u}\right]^{2}+\right. \\
\left.+\left[z_{u p}(1-2 \phi) \pm z_{p p}\right]^{2}+\left(z_{p p}^{2}+z_{u u}^{2}\right) / d^{2}\right\}, \\
\varphi_{1,2}(\phi)=p_{u}^{F}(1-2 \phi) \mp p_{p}^{F}=\pi\left[\left(n_{u} \mp n_{p}\right)-2 \phi n_{u}\right],
\end{gathered}
$$

where $n_{u}=N_{u} / L \equiv(N-2 M) / L$ and $n_{p}=N_{p} / L \equiv M / L$. Here and below the correlation functions are determined in the framework of the conformal field theory up to constants $A_{j}$.

For the Cooper-like pair-pair correlation function in the homogeneous mixed phase the set of quantum numbers is the following: $\Delta N_{p}=1, \Delta N_{u}=0, D_{p}=0, D_{u}= \pm 1 / 2$ and $n_{u}^{ \pm}=n_{p}^{ \pm}=0$. For $D_{u}=+1 / 2$ we have

$$
\begin{aligned}
& \Delta_{u}^{ \pm}=\frac{1}{8}\left[z_{u u}(1-2 \phi) \mp z_{u p} / d\right]^{2}, \\
& \Delta_{p}^{ \pm}=\frac{1}{8}\left[z_{u p}(1-2 \phi) \pm z_{u u} / d\right]^{2},
\end{aligned}
$$

and the exponent and the wave number of the phase of the correlation function are

$$
\begin{gathered}
\theta_{C P}(\phi)=\frac{1}{2}\left\{\left[z_{u u}(1-2 \phi)\right]^{2}+\right. \\
\left.+\left[z_{u p}(1-2 \phi)\right]^{2}+\left(z_{u p}^{2}+z_{u u}^{2}\right) / d^{2}\right\}, \\
\varphi_{C P}(\phi)=p_{F u}(1-2 \phi)=\pi\left[n_{u}-2 \phi n_{u}\right] .
\end{gathered}
$$


For $D_{u}=-1 / 2$ we have $\theta_{C P}(-\phi)$ and $-\varphi_{C P}(-\phi)$. The asymptote for the equal time pair-pair correlation function can then be written as

$$
\begin{aligned}
& \left\langle\psi_{\uparrow}^{\dagger}(x, 0) \psi_{\downarrow}^{\dagger}(x, 0) \psi_{\downarrow}(0,0) \psi_{\uparrow}(0,0)\right\rangle \approx \\
& \approx A_{C P}\left(\frac{\mathrm{e}^{-i \varphi_{C P}(\phi) x}}{x^{\theta} C P(\phi)}+\frac{\mathrm{e}^{i \varphi_{C P}(-\phi) x}}{x^{\theta} C P^{(-\phi)}}\right) .
\end{aligned}
$$

Consider now the long-distance asymptotic behavior of the density wave correlation function. The (spin or charge) density operator does not change the number of electrons, so that $\Delta N_{u}=\Delta N_{p}=0$. Hence, the $D_{u}$ and $D_{p}$ quantum numbers are integers. Several sets of backscattering quantum numbers and particle-hole quantum numbers are possible.

First, one can have $D_{u}=D_{p}=0$. If $n_{u}^{ \pm}=n_{p}^{ \pm}=0$ we obtain $\langle\hat{N}(x, 0) \hat{N}(0,0)\rangle \approx\langle\hat{N}\rangle^{2}$, where $\hat{N}$ is either the number of electrons or the magnetization. Next we consider one of $n^{ \pm}$equal to one and the other three equal to zero. For $\phi=0$ the contribution to the equal time correlation function falls off as $1 / x^{2}$. For $\phi \neq 0$ the density-density correlation function is proportional to

$$
A_{D W 00} \frac{\mathrm{e}^{-i \varphi_{D W 00}(\phi) x}}{x^{\theta} D W 00(\phi)}
$$

with the exponent and the wave number of the phase given by

$$
\theta_{D W 00}(\phi)=2+2 \phi^{2}\left[z_{u u}^{2}+z_{u p}^{2}\right], \varphi_{D W 00}(\phi)=-2 \pi \phi n_{u} .
$$

Then one can have $D_{u}=D_{p}= \pm 1$ and $n_{u}^{ \pm}=n_{p}^{ \pm}=0$, which case corresponds to the momentum transfer of $2 p_{F \uparrow}$ if $\phi=0$. For $\phi \neq 0$ the asymptote of the densitydensity correlation function is proportional to

$$
A_{D W 11}\left(\frac{\mathrm{e}^{-i \varphi} D W 11^{(\phi) x}}{x^{\theta_{D W 11}(\phi)}}+\frac{\mathrm{e}^{i \varphi_{D W 11}(-\phi) x}}{x^{\theta_{D W 11}(-\phi)}}\right),
$$

with the exponent and the wave number of the phase given by

$$
\begin{aligned}
\theta_{D W 11}(\phi)= & 2\left\{\left[z_{u u}(1-\phi)+z_{p u}\right]^{2}+\left[z_{u p}(1-\phi)+z_{p p}\right]^{2}\right\} \\
& \varphi_{D W 11}(\phi)=2 \pi n_{u}(1-\phi)+2 \pi n_{p}
\end{aligned}
$$

Finally, one can have $D_{u}=0$ and $D_{p}= \pm 1$ and $n_{u}^{ \pm}=n_{p}^{ \pm}=0$. This corresponds a momentum transfer of $2 p_{\downarrow}^{F}$ if $\phi=0$. For $\phi \neq 0$ the correlation functions are proportional to

$$
A_{D W 01}\left(\frac{\mathrm{e}^{-i \varphi_{D W 01}(\phi) x}}{x^{\theta_{D W 01}(\phi)}}+\frac{\mathrm{e}^{i \varphi_{D W 01}(-\phi) x}}{x^{\theta_{D W 01}(-\phi)}}\right)
$$

with the exponent and the wave number of the phase given by

$$
\begin{gathered}
\theta_{D W 01}(\phi)=2\left\{\left[-z_{u u} \phi+z_{p u}\right]^{2}+\left[-z_{u p} \phi+z_{p p}\right]^{2}\right\}, \\
\varphi_{D W 01}(\phi)=-2 \pi n_{u} \phi+2 \pi n_{p} .
\end{gathered}
$$

In the gapped homogeneous phase one has only Fermi sea for pairs, which do not carry spin, and, hence, they are not affected by the SO coupling. For $H<H_{c}$ the Green function decays exponentially. For the gapped case we have $z_{p p}=\xi_{p p}\left(v_{0}\right)$ and $D_{p}$ is an integer. The former is determined from the solution of the equation

$$
\xi_{p p}(\Lambda)=1-\int d z f(z-\Lambda, \eta) \xi_{p p}(z)
$$

and the conformal dimensions are given by

$$
\Delta_{p}^{ \pm}=n_{p}^{ \pm}+\left[z_{p p} D_{p} \pm \Delta N_{p} /\left(2 z_{p p}\right)\right]^{2}
$$

For the single-electron Green function we have to break up a Cooper pair. Since the spectrum of unpaired particles is gapped, Green's functions decay exponentially with the distance.

For the Cooper-like pairs we consider $\Delta N_{p}=1, D_{p}=0$ and $n_{p}^{ \pm}=0$. The conformal dimensions are then $\Delta_{p}^{ \pm}=\left(8 z_{p p}^{2}\right)^{-1}$, and the asymptote for the pair-pair correlation function can be written as

$$
\left\langle\psi_{\uparrow}^{\dagger}(x, 0) \psi_{\downarrow}^{\dagger}(x, 0) \psi_{\downarrow}(0,0) \psi_{\uparrow}(0,0)\right\rangle \approx A_{C P} \frac{1}{x^{\theta} C P},
$$

where the exponent is $\theta_{C P}(\phi)=1 /\left(2 z_{p p}\right)^{2}$.

Finally, for the density wave correlation function two contributions are possible. For the first one $\left(D_{p}=0\right)$ the correlation function is proportional to $x^{-2}$, and the second one with momentum transfer of $2 p_{F}, D_{p}= \pm 1$, $n_{p}^{ \pm}=0$ is proportional to $x^{-\theta} D W 01$. The exponent of the phase in this case is $\theta_{D W 01}=2 z_{p p}^{2}$. Notice that in the gapped phase, which has no spin-imbalance, oscillations in the Cooper-pair correlation function arise for $\phi \neq 0$.

For the values of $\mu$ in the gapped phase (for $H<H_{c}$ ) and in the mixed phase $H_{c} \leq H \leq H_{s}$ for the values of $\mu$ and $H$, at which spatially inhomogeneous distributions of unbound electron excitations and spin-singlet pairs take place, the behavior of algebraically decaying correlations functions is more complicated.

For $H>H_{s}$ the band of Cooper-like pairs is empty and all the unpaired electrons are spin-polarized. The unbound electrons are now non-interacting and all correlation functions are those of free particles modified by the SO interaction phase. 


\section{Finite temperatures}

At finite but low temperatures the main contribution to thermodynamics of the system comes from low-energy (gapless) excitations. Namely those excitations contribute mostly to the low-temperature dependence of the charge susceptibility (compressibility), magnetic susceptibility, and the specific heat. Contributions from other (gapped) eigenstates is exponentially small. At low temperatures the second order phase transitions, mentioned above, manifest themselves as $1 / \sqrt{T}$ features corresponding to the van Hove singularities of the edges of the bands. At the critical values of $\theta$ the behavior shows $T^{-3 / 4}$ features. At nonzero temperatures in the thermodynamic limit the same exponents determine the temperature behavior of correlation functions with the obvious replacement, $\left(x \pm i v_{j} t\right) \rightarrow$ $\rightarrow v_{j} \sinh \left(\pi T\left[x \pm i v_{j} t\right) / v_{j}\right) / \pi T$.

\section{Summary}

The novel Bethe-ansatz integrable model of correlated electrons on the one-dimensional lattice with nearest- and next-nearest neighbor hoppings and interactions, equivalent to the zig-zag lattice with the spin-orbit coupling is introduced. The model describes the supersymmetric $t$ - $J$-model with the "easy-axis" (Ising-like) type of the anisotropy on the geometrically frustrated lattice. As other one-dimensional quantum models, it manifests the most interesting behavior in the ground state, where the number of quantum phase transitions, governed by the band filling and the external magnetic field, take place. The Ising type of the anisotropy gives rise to a gap for low-energy unbound electron states at small values of the magnetic field, therefore the model is reminiscent of the type-II superconductor. Namely, for small fields only spin-singlet Cooper-like pairs are gapless. For larger values of the field both pairs and unbound electron states are gapless. Finally for larger field the system is in the spinpolarized phase. For the large value of the parameter, which describes the inter-chain (NNN) coupling and hopping, the model manifests additional quantum phase transitions to phases with inhomogeneously distributed charge and/or spin densities, in the simplest case of the commensurate structures, analogous to the CDW or SDW states, or FFLO ordering [33]. At low temperatures, as usually, quantum phase transitions reveal themselves in square root in temperature features in the behavior of the magnetic and charge susceptibility and the specific heat. The spin-orbit coupling manifests itself in the finite size corrections, i.e., in the behavior of exponents and phases of correlation functions for some gapless states. The features of the model can be used in the description of real quasi-one-dimensional correlated electron systems, in particular, zig-zag chains of magnetic ions on a surface of a superconductor, important for the search for Majorana zero modes. We admit that some features of exactly solvable one-dimensional models are far from being ob- served experimentally. However those non-realistic features are known and simple to recognize.

\section{Acknowledgements}

The support from the DFG via SFB 1143 is acknowledged.

1. N.D. Mermin and H. Wagner, Phys. Rev. Lett. 17, 1133 (1966).

2. A.A. Zvyagin, Finite Size Effects in Correlated Electron Models: Exact Results, Imperial College Press, London (2005).

3. See, e.g., Frustrated Spin Systems, H.T. Diep (ed.), World Scientific, Singapore (2004); Highly Frustrated Magnetism, C. Lacroix, P. Mendels, and F. Mila (eds.), Springer, Berlin (2010); A.P. Ramirez, Annu. Rev. Mater. Sci. 24, 453 (1994); R. Moessner, J. Phys.: Conf. Ser. 145, 012001 (2009); R. Moessner and A.R. Ramirez, Physics Today 59, 24 (2006); C. Castelnovo, R. Moessner, and S.L. Sondhi, Ann. Rev. Condens. Mater. Phys. 3, 35 (2012); A.A. Zvyagin, Fiz. Nizk. Temp. 39, 1159 (2013) [Low Temp. Phys. 39, 901 (2013)].

4. See, e.g., P.A. Lee, Science 321, 1306 (2008); L. Balents, Nature 464, 199 (2010); Y. Zhou and P.A. Lee, Phys. Rev. Lett. 106, 056402 (2011); L. Savary and L. Balents, Rep. Progr. Phys. 80, 016502 (2017).

5. See, e.g., C.K. Majumdar and D.K. Ghosh, J. Math. Phys. 10, 1388 (1969); K. Okamoto and K. Nomura, Phys. Lett. A 169, 433 (1992); V.Y. Popkov and A.A. Zvyagin, Phys. Lett. A 175, 295 (1993); M. Matsuda and K. Katsumata, J. Magn. Magn. Mater. 140-145, 1671 (1995); S.R. White and I. Affleck, Phys. Rev. B 54, 9862 (1996); H. Kikuchi, H. Hagasawa, Y. Ajiro, T Asano, and T. Goto, Physica B 284-288, 1631 (2000); A.A. Zvyagin, J. Phys. A 34, R21 (2001); K. Okunishi, Progr. Theor. Phys. Suppl. 145, 119 (2002); N. Maeshima, M. Hagiwara, Y. Narumi, K. Kindo, T.C. Kobayasi, and K. Okunishi, J. Phys.: Condens. Matter 15, 3607 (2003); M. Hase, K. Ozawa, and N. Shinya, Phys. Rev. B 68, 214421 (2003); T. Masuda, A. Zheludev, A. Bush, M. Markina, and A. Vasiliev, Phys. Rev. Lett. 92, 177201 (2004); T. Masuda, A. Zheludev, A. Bush, M. Markina, and A. Vasiliev, Phys. Rev. Lett. 94, 039706 (2005); S.-L. Drechsler, J. Málek, J. Richter, A.S. Moskvin, A.A. Gippius, and H. Rosner, Phys. Rev. Lett. 94, 039705 (2005); A.A. Gippius, E.N. Morozova, A.S. Moskvin, A.V. Zalessky, A.A. Bush, M. Baenitz, H. Rosner, and S.-L. Drechsler, Phys. Rev. B 70, 020406(R) (2004); L. Capogna, M. Mayr, P. Horsch, M. Raichle, R.K. Kremer, M. Sofin, A. Maljuk, M. Jansen, and B. Keimer, Phys. Rev. B 71, 140402(R) (2005); S.-L. Drechsler, O. Volkova, A.N. Vasiliev, N. Tristan, J. Richter, M. Schmitt, H. Rosner, J. Málek, R. Klingeler, A.A. Zvyagin, and B. Büchner, Phys. Rev. Lett. 98, 077202 (2007), A.A. Zvyagin and S.-L. Drechsler, Phys. Rev. B 78, 014429 (2008); A. Vasiliev, O. Volkova, E. Zvereva, and M. Markina, NPJ Quantum Materials 3, 18 (2018); N. Blanc, J. Trinh, L. Dong, 
X. Bai, A.A. Aczel, M. Mourigal, L. Balents, T. Siegrist, and A.P. Ramirez, Nature Phys. 14, 273 (2018).

6. Y.-W. Son, M.L. Cohen, and S.G. Louie, Nature 444, 347 (2006).

7. E. Berg, T.H. Geballe, and S.A. Kivelson, Phys. Rev. B 76, 214505 (2007).

8. A. Poddubny, A. Miroshnichenko, A. Slobozhayuk, and Y. Kivshar, Act Photonics 1, 1001 (2014); L. Lu, J.D. Joannopoulos, and M. Soljacic, Nat. Photon. 8, 821 (2014).

9. G.E. Astrakharchik, G. Morigi, G. De Chiara, and J. Boronat, Phys. Rev. A 78, 063622 (2008); E. Shimshoni, G. Morigi, and S. Fishman, Phys. Rev. Lett. 106, 010401 (2011); D. Rossini, V. Lante, A. Parola, and F. Becca, Phys. Rev. B 83, 155106 (2011); G. Sun, G. Jackeli, L.Santos, and T. Vekua, Phys. Rev. $B$ 86, 155159 (2012); F. Cartarius, A. Minguzzi, and G. Morigi, Phys. Rev. A 95, 063603 (2017).

10. S. Nadj-Perge, Y.K. Drozdov, J. Li, H. Chen, S. Jeon, J. Seo, A.H. Macdonald, B.A. Bernevig, and A. Yazdani, Science 346, 602 (2014).

11. I.K. Drozdov, A. Alexandradinata, S. Jeon, S. Nadj-Perge, H. Ji, R.J. Cava, B.A. Bernevig, and A. Yazdani, Nature Phys. 10, 664 (2014).

12. X. Xi, Z. Wang, W. Zhao, J.-H. Park, K.T. Law, H. Berger, L. Forró, J. Shan, and K.F. Mak, Nature Phys. 12139 (2016).

13. G. Dresselhaus, Phys. Rev. B 100, 580 (1955); Y.A. Bychkov and E.I. Rashba, J. Phys. C 17, 6039 (1984).

14. A.A. Zvyagin, Phys. Rev. B 95, 165141 (2017).

15. M.Z. Hasan and C.L. Kane, Rev. Mod. Phys. 82, 3045 (2010); X.-L. Qi and S.-C. Zhang, Rev. Mod. Phys. 83, 1057 (2011).

16. R.M. Lutchyn, J.D. Sau, and S. Das Sarma, Phys. Rev. Lett. 105, 077001 (2010); Y. Oreg, G. Refael, and F. von Oppen, Phys. Rev. Lett. 105, 177002 (2010).

17. For a review see, e.g., I. Bloch, Nature Phys. 1, 23 (2005) and references therein.

18. Y.A. Liao, A.S.C. Rittner, T. Paprotta, W.H. Li, G.B. Partridge, R.G. Hulet, S.K. Baur, and E.J. Mueller, Nature (London) 467, 567 (2010).

19. G. Orso, Phys. Rev. Lett. 98, 070402 (2007); H. Hu, X.-J. Liu, and P.D. Drummond, Phys. Rev. Lett. 98, 070403 (2007); P. Schlottmann and A.A. Zvyagin, Phys. Rev. B 85, 205129 (2012); A.A. Zvyagin and P. Schlottmann, Phys. Rev. B 88, 205127 (2013); P. Schlottmann and A.A. Zvyagin, Nucl. Phys. B 892, 269 (2015).

20. M. Olshanii, Phys. Rev. Lett. 81, 938 (1998); T. Bergeman, M.G. Moore, and M. Olshanii, Phys. Rev. Lett. 91, 163201 (2003).

21. For a recent review see, e.g., P. Schlottmann and A.A. Zvyagin, Mod. Phys. Lett. B 26, 1230009 (2012) and references therein.

22. X.-J. Liu, M.F. Borunda, X. Liu, and J. Sinova, Phys. Rev. Lett. 102, 046402 (2009); Y.-J. Lin, R.L. Compton, K. Jiménez-García, J.V. Porto, and I.B. Spielman, Nature 426, 628 (2009); Y.-J. Lin, K. Jiménez-García, and I.B. Spielman, Nature 471, 83 (2011); Y.-J. Lin, R.L. Compton, K. Jiménez-
García, W.D. Phillips, J.V. Porto, and I.B. Spielman, Nature Phys. 7, 531 (2011); M. Chapman and C. Sá de Melo, Nature 471, 41 (2011); M. Aidelsburger, M. Atala, S. Nascimbéne, S. Trotzky, Y.-A. Chen, and I. Bloch, Phys. Rev. Lett. 107, 255301 (2011); R.A. Williams, L.J. LeBlanc, K. JiménezGarcía, M.C. Beeler, A.R. Perry, W.D. Phillips, and I.B. Spielman, Science 335, 314 (2012); J.-Y. Zhang, S.-C. Ji, Z. Chen, L. Zhang, Z.-D. Du, B. Yan, G.-S. Pan, B. Zhao, Y.-J. Deng, H. Zhai, S. Chen, and J.-W. Pan, Phys. Rev. Lett. 109, 115301 (2012).

23. P. Wang, Z.-Q. Yu, Z. Fu, J. Miao, L. Huang, S. Chai, H. Zhai, and J. Zhang, Phys. Rev. Lett. 109, 095301 (2012); L.W. Cheuk, A.T. Sommer, Z. Hadzibabic, T. Yefsah, W.S. Bakr, and M.W. Zwierlein, Phys. Rev. Lett. 109, 095302 (2012).

24. Y. Meir, Y. Gefen, and O. Entin-Wohlman, Phys. Rev. Lett. 63, 798 (1989).

25. A. Foerster and M. Karowski, Nucl. Phys. B 396, 611 (1993); A. Foerster and M. Karowski, Nucl. Phys. B 408, 512 (1994); R.Z. Bariev, Phys. Rev. B 49, 1474 (1994).

26. C.K. Lai, J. Math. Phys. 15, 167 (1974); B. Sutherland, Phys. Rev. B 12, 3795 (1975); P. Schlottmann, Phys. Rev. B 36, 5177 (1987).

27. A.A. Zvyagin, Fiz. Nizk. Temp. 40, 83 (2014) [Low Temp. Phys. 40, 65 (2014)].

28. A.A. Zvyagin, Phys. Rev. B 52, 15050 (1995)

29. A.A. Zvyagin, Phys. Rev. B 57, 1035 (1998).

30. P. di Francesco, P. Mathieu, and D. Sènèchal, Conformal Field Theory, Springer-Verlag, New York (1997).

31. V.E. Korepin, N.M. Bogoliubov, and A.G. Izergin, Quantum Inverse Scattering Method and Correlation Functions, Cambridge University Press, Cambridge (1993).

32. A.A. Zvyagin, Phys. Rev. B 86, 085126 (2012); A.A. Zvyagin and P. Schlottmann, Phys. Rev. B 88, 205127 (2013).

33. P. Fulde and A. Ferrell, Phys. Rev. A 135, 550 (1964); A. Larkin and Y.N. Ovchinnikov, Zh. Eksp. Teor. Fiz. 47, 1136 (1964) [Sov. Phys. JETP 20, 762 (1965)].

Корельовані електрони на гратці типу зигзаг зі спін-орбітальною взаємодією: точне рішення

\section{А.А. Звягін}

Вивчаєтся модель корельованих електронів на геометрично фрустрованій одновимірній гратці зі спін-орбітальною взаємодією. Одержано точне вирішення методом анзаца Бете. Взаємодія на гратці типу зигзаг може призвести до появи несорозмірних структур для великих значень фруструючих взаємодій. Спін-орбітальна взаємодія призводить до поведінки кореляційних функцій, які нагадують особливості типу Фульде-Ферреля-Ларкіна-Овчинникова в реальних надпровідниках другого роду.

Ключові слова: інтегрована модель корельованих електронів, геометрична фрустрація, спін-орбітальна взаємодія. 


\section{Коррелированные электроны на решетке типа зигзаг со спин-орбитальным взаимодействием: точное решение}

\section{А.А. Звягин}

Изучается модель коррелированных электронов на геометрически фрустрированной одномерной решетке со спинорбитальным взаимодействием. Получено точное решение методом анзаца Бете. Взаимодействие на решетке типа зигзаг может привести к появлению несоизмеримых структур для больших значений фрустрирующих взаимодействий. Спинорбитальное взаимодействие приводит к поведению корреляционных функций, напоминающих особенности типа ФульдеФерреля-Ларкина-Овчинникова в реальных сверхпроводниках второго рода.

Ключевые слова: интегрируемая модель коррелированных электронов, геометрическая фрустрация, спин-орбитальное взаимодействие. 